\title{
Hybrid Particle Swarm Optimization-Fuzzy Inference System for Premature Atrial Contraction Detection
}

\author{
Nuryani Nuryani \\ Department of Physics, University of Sebelas Maret Surkarta, Indonesia \\ email:nuryani@mipa.uns.ac.id \\ Iwan Yahya \\ Department of Physics, University of Sebelas Maret Surkarta, Indonesia \\ Anik Lestari \\ Faculty of Medicine, University of Sebelas Maret, Surakarta, Indonesia
}

\begin{abstract}
This article presents a new technique to detect a premature atrial contraction (PAC). The technique employs a hybrid of particle swarm optimization (PSO) and fuzzy inference system (FIS), and is called PSO-FIS. In the detection electrocardiographic features are used for the inputs of PSO-FIS. In PSO-FIS, a PSO is used to find the optimal parameters of the FIS. A Gaussian function is employed for the fuzzification part of the FIS. The inputs of the FIS are the interval between two consecutive electrocardiographic R waves and the accumulation of the amplitudes around the $\mathrm{P}$ waves. Using clinical data, the technique performs well for PAC detection with $81.93 \%$, $82.27 \%$ and $82.26 \%$ respectively.
\end{abstract}

Keywords: Particle swarm optimization, fuzzy inference system, premature atrial contraction, electrocardiogram

\section{Introduction}

A Premature atrial contraction (PAC) is an abnormal electrical heart beat starting from the atrial chambers of the heart and alters the heart normal rhythm. PAC is also called atrial premature complexes (APC). It happens when the atria of the heart depolarizes prematurely which could trigger a premature electrocardiographic $\mathrm{P}$ wave. $\mathrm{PAC}$ is dangerous in patients with heart diseases as it is associated with lifethreatening.

This paper proposes a strategy for detecting PAC. A swarm based fuzzy inference system (SFIS) and electrographic features are investigated. SFIS is a fuzzy inference system optimized using particle swarm optimization (PSO). Fuzzy system has shown good performances for various applications, including for medical problems. Two electrocardiographic features investigated. The first is the interval between two consecutive $\mathrm{R}$ waves, which is called RR in this this article. The second is the accumulative amplitudes around the electrocardiographic $\mathrm{P}$ wave, which is called $\mathrm{P}$ amplitudes (PA). RR interval in patient with PAC problem is shorter than the normal one. In addition, the amplitudes around the $\mathrm{P}$ wave might be higher in patient with PAC than in the normal one, as in the patient the $\mathrm{T}$ wave comes closely to $\mathrm{P}$ wave.

The rest of this article is presented as follows. The technique of the PAC detection is presented in Section II. Section III and IV presents the experimental results and discussion, respectively. Section V presents the conclusion.

\section{Method}

The proposed PAC detection technique is presented in Fig 1. Three main parts of the technique are: inputs, SFIS and output. The inputs are RR and PA. SFIS is a FIS optimized using PSO. It is used to classify an input 
is belong to PAC or normal. Thus the output of the technique is one of two conditions: PAC or normal.

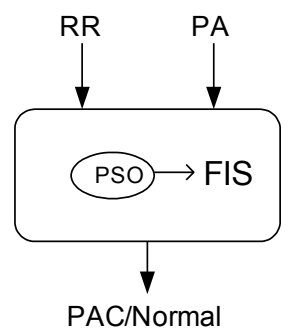

Fig. 1. than one line then it is centered

\subsection{Inputs}

The inputs of the detection technique are RR and RA. $\mathrm{RR}$ is the interval of two consecutive R-peaks. The positions of $\mathrm{R}$ peaks are defined by Physionet. Therefore, what we need is to calculate the intervals (Fig. 2). RA feature is defined as the sum of amplitudes around the electrocardiographic $\mathrm{R}$ waves (Fig 3). To reduce the effects of electrocardiographic varieties between patient, a normalization $0-1$ is conducted.

\subsection{Fuzzy Inference System (FIS)}

Fuzzy inference system or FIS is a process to map a given input to an output using fuzzy logic. The mapping provides a system from which decisions can be made. FIS involves three main parts: fuzzification, fuzzy IFTHEN rules, and defuzzification..

$$
\mu_{N_{i}^{l}}\left(x_{i}\right)=e^{-\left(x-m_{i}^{l}\right)^{2} / 2 \sigma_{i}^{l}}
$$

\subsubsection{Fuzzification}

The first component of FIS is fuzzification. It converts a real-world space (the inputs) to a fuzzy space ${ }^{l}$, as illustrated in Fig. 5. The inputs of fuzzification are RR and RA, as defined beforehand. The outputs of fuzzification are the membership function degrees associated with the inputs.
The fuzzification employs a Gaussian membership function, which can be expressed as ${ }^{2}$

where $x_{i}$ is the inputs; $m$ and $\sigma$ are mean and standard deviation of the Gaussian membership function, respectively; $l$ represents membership function terms.

\subsubsection{Fuzzy IF-THEN rules}

The second component of FIS is fuzzy IF-THEN rules. The rules map a fuzzy input to a fuzzy output using an IF-THEN rule. Each rule consists of two parts: antecedence ('IF') and its consequence ('THEN'). The rule is provided as the following ${ }^{3}$ :

Ruler: IF $x_{1}$ is $N_{1}^{l}$ AND $x_{2}$ is $N_{2}^{l}$ THEN $\gamma$ is $\beta_{r}(2)$

where $N_{i}^{l}(i=1,2, \ldots, n)$ is fuzzy term of the associated input $x_{j}$. Rule $r=1,2, \ldots, \tau$ denotes the rule number; $\tau$ denotes the number of rules. The number of rule is $v^{n} ; v$ is the number of membership functions and $n$ is the number of inputs. $\beta_{r}$ is a fuzzy singleton to be optimized.

\subsubsection{Defuzzification}

Defuzzification is the last part of FIS. It converts the output of the fuzzy rules into a real world value ${ }^{l}$. Defuzzification result is given by

$$
\eta=\sum_{r=1}^{\tau} \varsigma_{r} \beta_{r}
$$

Where

$$
\varsigma_{r}=\frac{\mu_{N_{i}^{l}}\left(x_{1}\right) \times \mu_{N_{2}^{l}}\left(x_{2}\right)}{\sum_{r=1}^{\tau} \mu_{N_{i}^{l}}\left(x_{1}\right) \times \mu_{N_{2}^{l}}\left(x_{2}\right)}
$$

Optimization of FIS using PSO tunes the mean $m$, the standard deviation $\sigma$ and the fuzzy singleton $\beta$ which is the output of IF-THEN rules. Thus, during the training of FIS, these parameters are tunes automatically to find the optimal value. 


\subsection{Particle Swarm Optimization (PSO)}

As mentioned beforehand that in this article PSO is employed to optimize FIS parameters. These optimization is conducted to optimizes FIS in order that it could perform well. Essentially, PSO performs optimization using an evolutionary technique. The technique is based on movement of swarms and is inspired by social behavior of bird flocking and fish schooling ${ }^{4}$.

The algorithm of PSO expressed by pseudo code can presented in Fig. 6. The initial step is to define a swarm $Z(t)$. The swarm represents the FIS parameter defined beforehand. A fitness function is defined and is then evaluated. Essentially, the objective of the optimization is to minimize a fitness function $f(Z(t))$ iteratively. The swarm evolves from the first iteration until the end of iteration.

The swarm consisting particles fly with position and velocity updated every iteration. The position $y$ and velocity $v$ of particles are defined as the following:

$$
y(t)=y(t-1)+v(t)
$$

and

$$
v(t)=q\left(\varphi v(t-1)+c_{1} r_{1}\left(z_{p}-z(t-1)\right)+c_{2} r_{2}\left(z_{g}-z(t-1)\right)\right)
$$

where $z_{p}$ is the position of a particle in the previous iteration with the best value, and $z_{g}$ is the best particle position among the all particles. $r_{1}$ and $r_{2}$ are random values in the range [ 01$]$, and $\varphi$ is inertia weight factor. $c_{1}$ and $c_{2}$ are acceleration constants. $q$ is a constriction factor to ensure the optimization to be converged, but not prematurely.

The objective of the optimization is to minimize the following equation:

$$
f=-\left(S e_{t}+S p_{t}\right)
$$

where $S e_{t}$ and $S p_{t}$ are the sensitivity and specificity, respectively. Sensitivity is defined as the ratio of the correct detection of PAC to the actual number of PAC cases; specificity is defined as the ratio of the correct detection of normal to the actual number of normal cases.

\section{Results}

The proposed technique to detect PAC has been developed and examined with clinical data. The data is collected from MIT-BIH arrhythmia database ${ }^{5}$. The clinical data are the electrocardiograms of six patients.
The patients are with the record numbers of 100, 200, 202 and 213.

Electrocardiogram with PAC is presented in Fig . This figure presents normal ECG (the red one) and ECG with PAC (the blue one), obtained from clinical data. Each ECG includes two QRS waves and the waves between the two waves. Regarding electrocardiographic features for PAC detection, this article concerns the RR interval of ECG. It can be seen from the figure, ECG with PAC has RR interval shorter than the normal one. In PAC, the $\mathrm{P}$ wave comes too early or prematurely which shorten the area between two QRS waves. This phenomenon is important feature for the PAC detection proposed in this article.

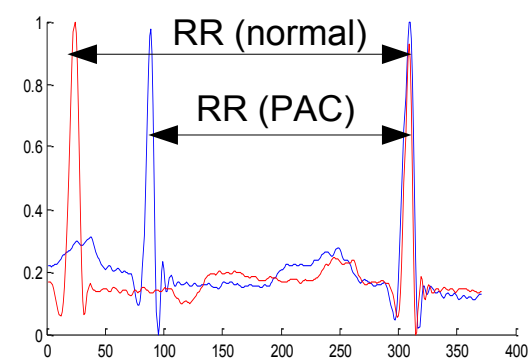

Another important electrocardiographic feature for PAC is the $\mathrm{P}$ wave coming too early or premature $\mathrm{P}$ wave. The premature $\mathrm{P}$ wave might be very close or even overlap to electrocardiographic $\mathrm{T}$ wave. Because of this, there is an area on an electrocardiogram where the amplitudes are higher than the normal. This area is around the $\mathrm{T}$ wave. Thus, this area has amplitudes higher than the normal. In this article, this area is called PA. This issue can be presented in Fig

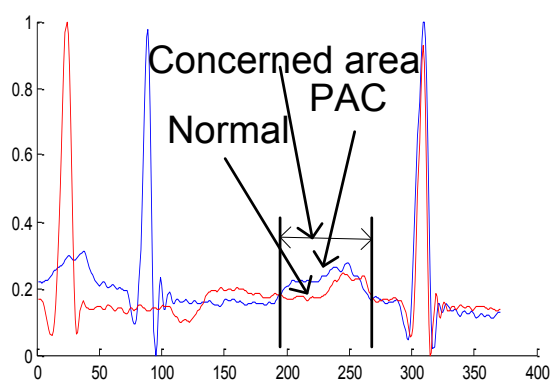

Please make sure that no page numbers appear in your paper. Sections, sub-sections and sub-subsections are numbered in Arabic. Use double spacing before all section headings and single spacing after section headings. Flush left all paragraphs that follow after section headings. 
The detection performances of PSO-FIS with different inputs are presented in Table I. Three variations of the inputs are employed: RR, PA and RR-PA which are called PSO-FIS1, PSO-FIS2 and PSO-FIS3. The performances are measured in terms of sensitivity $(\mathrm{Se})$, specificity $(S p)$ and accuracy $(A c)$.

Table 1. The performances of PSO-FIS with different inputs

\begin{tabular}{ccccccc}
\hline \multirow{2}{*}{ Method } & \multicolumn{3}{c}{ Training } & \multicolumn{3}{c}{ Testing } \\
& Se (\%) & Sp (\%) & Ac (\%) & Se (\%) & Sp (\%) & Ac (\%) \\
\hline PSO-FISp & 64.46 & 3.61 & 64.64 & 59.64 & 64.91 & 64.75 \\
PSO-FISr & 75.90 & 77.67 & 77.62 & 76.51 & 77.02 & 77.01 \\
PSO-FISrp & 88.55 & 81.55 & 81.76 & 81.93 & 82.27 & 82.26 \\
\hline
\end{tabular}

The performance of PSO-FISp which is the PSO-FIS with the input of PA performs the worst among the three approaches. The performance becomes higher by changing the input by RR. The performance becomes higher again when the inputs are both RR and PA.

This result implies that $\mathrm{RR}$ is more significant to positively contribute to provide a good performance of the detection. This result confirms that RR interval is the important indication of PAC, where PAC RR interval is shorter in patient with $\mathrm{PAC}$ than the normal one.

Another feature is PA which is the total of amplitudes around the $\mathrm{P}$ wave. The result shows that the performance PSO-FIS with the input of PA is lower than which the input of RR. But this PA provides positive contribution to improve the performance as adding PA to the input provide higher performance.

\section{Conclusion}

This article has proposed and investigated a method for detecting premature atrial contraction (PAC). The method employs a fuzzy inference system (FIS) optimized by a particle swarm optimization (PSO), called PSO-FIS. The inputs examined for PSO-FIS are the electrocardiographic RR interval and the accumulation of the amplitudes around the $\mathrm{P}$ wave. Investigating PSO-FIS with clinical data shows that this method could detect PAC with sensitivity, septicity and accuracy of $81.93 \%, 82.27 \%$ and $82.26 \%$ respectively.

\section{References}

1. G. Feng, "A survey on analysis and design of model-based fuzzy control systems," IEEE Transactions on Fuzzy Systems vol. 14, pp. 676-697, 2006.

2. S. Feng, H. Li, and D. Hu, "A new training algorithm for HHFNN based on Gaussian membership function for approximation," Neurocomputing, vol. 72, pp. 1631-1638, 2009.

3. C. Xiaoguang and J. H. Lilly, "Evolutionary design of a fuzzy classifier from data," IEEE Transactions on Systems, Man, and Cybernetics, Part B: Cybernetics, , vol. 34, pp. 1894-1906, 2004.

4. J. Kennedy and R. Eberhart, "Particle swarm optimization," in IEEE International Conference on Neural Networks 1995, pp. 1942-1948.

5. G. B. Moody and R. G. Mark, "The impact of the MITBIH arrhythmia database," IEEE Engineering in Medicine and Biology Magazine, vol. 20, pp. 45-50, 2001. 